\title{
Integrating epigenetic, genetic, and phenotypic data to uncover gene-region associations with triglycerides in the GOLDN study
}

\author{
Razvan G. Romanescu ${ }^{1 *}$, Osvaldo Espin-Garcia ${ }^{1,2}$, Jin Ma ${ }^{1}$ and Shelley B. Bull ${ }^{1,2}$ \\ From Genetic Analysis Workshop 20 \\ San Diego, CA, USA. 4 - 8 March 2017
}

\begin{abstract}
Background: There has been significant interest in investigating genome-wide and epigenome-wide associations with lipids. Testing at the gene or region level may improve power in such studies.

Methods: We analyze chromosome 11 cytosine-phosphate-guanine (CpG) methylation levels and single-nucleotide polymorphism (SNP) genotypes from the original Genetics of Lipid Lowering Drugs and Diet Network (GOLDN) study, aiming to explore the association between triglyceride levels and genetic/epigenetic factors. We apply region-based tests of association to methylation and genotype data, in turn, which seek to increase power by reducing the dimension of the gene-region variables. We also investigate whether integrating 2 omics data sets (methylation and genotype) into the triglyceride association analysis helps or hinders detection of candidate gene regions.
\end{abstract}

Results: Gene-region testing identified $1 \mathrm{CpG}$ region that had been previously reported in the GOLDN study data and another 2 gene regions that are also associated with triglyceride levels. Testing on the combined genetic and epigenetic data detected the same genes as using epigenetic or genetic data alone.

Conclusions: Region-based testing can uncover additional association signals beyond those detected using singlevariant testing.

\section{Background}

Many authors have called for greater use of gene-based approaches to detect candidate regions at the genome-wide discovery stage, raising concerns that exclusive marginal single-variable testing may miss more complex associations. For example, Yoo et al. [1] report that region tests can be more sensitive to genetic architectures with multiple causal components, and find that reduced-dimension test statistics, such as that proposed by Gauderman et al. [2], can improve power compared to tests in full multivariable regressions. To some extent, this argument also applies to genome-wide epigenetic studies, but conclusive evidence is

\footnotetext{
* Correspondence: razvan@lunenfeld.ca

${ }^{1}$ Lunenfeld-Tanenbaum Research Institute, Sinai Health System, 600

University Ave, Toronto, ON M5G 1X5, Canada

Full list of author information is available at the end of the article
}

lacking for it. Specification of the constituent variables for a gene region, however, is a major challenge in implementation for both genetic and epigenetic gene-region modeling, and is critical for integration of the 2 data sources when the molecular technology platforms differ.

In their investigation of epigenome-wide association of fasting blood lipids in the Genetics of Lipid Lowering Drugs and Diet Network (GOLDN) study, Irvin et al. [3] model the percentage methylation separately at each individual cytosine-phosphate-guanine $(\mathrm{CpG})$ site as a function of triglyceride levels. They report genome-wide significant associations of $4 \mathrm{CpG}$ sites in intron 1 of the CPT1A gene located on chromosome 11. In this article, we apply gene-region association methods to the original chromosome 11 epigenetic data from the GOLDN study [3], supplemented with chromosome 11 genome-wide association 
study single-nucleotide polymorphism (SNP) data available in a common subset of individuals. Our aims are to explore the association between baseline triglyceride (TG) levels and genetic/epigenetic factors using gene-region analysis methods, and to investigate 1 approach to integration of 2 omics data types (SNP genotype and CpG methylation) by comparing the integrated approach with separate analyses.

\section{Methods}

\section{Data}

We take the phenotype to be log-transformed triglyceride (lnTG) using averaged TG measurements before treatment with fenofibrate. To investigate genetic association with the phenotype, we convert the Affymetrix platform SNP genotypes to allele counts coded as 0,1 , or 2 . In total, we consider 36,796 SNPs on chromosome 11. The methylation data for the same chromosome consists of 28,285 CpG sites in total. The number of participants from the original study with sufficiently complete epigenetic data are 995. Of the 995 participants, 717 had genotype information as well.

\section{Specification of gene regions}

Sets of CpG sites and SNPs corresponding to each gene region were obtained using the GENCODE [4] annotation file bundled with LocusZoom standalone software [5], and expanding each genetic region by $20 \mathrm{~kb}$ before the start, and after the end, of the annotated base-pair positions. This was done to include any possibly related functional SNPs or CpG sites from each gene neighborhood. In all, we defined 2621 gene regions on chromosome 11. The number of component variables per gene region ranged from 2 to 544 for SNPs and from 2 to 372 for CpG sites. For computational reasons, we excluded 6 genetic regions (Metazoa_SRP, SNORA1, SNORA7, U3, $Y_{-} R N A$, snoU13) that had more than 2000 CpG or SNPs from the subsequent gene-region regression analysis.

\section{Single $\mathrm{CpG}$ epigenetic association}

To investigate association between TGs and methylation on chromosome 11, we regress percentage methylation on $\operatorname{lnTG}$ measurements as in Irvin et al. [3], and include age, study site, sex, and cell purity as fixed effects, and family as a random effect. TG values are first averaged over the measurements pretreatment (at most 2 per participant), as this yields the most complete data set (995 cases). Cell purity variables estimated as the top 4 principal components of the methylation data, are included as fixed effects. The model reads (in $\mathrm{R}$ notation):

$$
\begin{gathered}
\mathrm{CpG} \sim \ln (\mathrm{TG})+\text { age }+ \text { center }+ \text { sex }+P C 1^{G} \\
+P C 2^{G}+P C 3^{G}+P C 4^{G}
\end{gathered}
$$

with a random effect for GPEDID, the family ID from the pedigree file, used to account for familial correlation. Here, the superscript $G$ indicates that the principal component $(P C)$ for cell purity is computed globally for chromosome 11 . We note 2 differences from the original GOLDN study. First, the chromosome 11 methylation data we use to calculate cell purity $P C s$ ) has 28,285 CpG sites, whereas in Irvin et al. [3] the same procedure was based on 461,281 CpG sites from the whole genome (after quality control). Second, model (1) assumes a common correlation among members of the same family, whereas the original analysis used the kinship coefficient to define the correlation of random effects. Our approach is much faster computationally as it uses the lmer function rather than Imekin (as in Irvin et al. [3]). We also confirmed the fit using the kinship coefficient, but note that the $p$ values obtained using model (1) already match those in the original paper fairly closely.

\section{Gene-region testing of SNP genetic and CpG epigenetic association}

To assess the value of integrating the 2 types of data in detecting gene regions associated with the TG phenotype, we regress $\operatorname{lnTG}$ on SNP-derived and CpG-derived predictors. We employ the method of Gauderman et al. [2], which computes the PCs of the regressors, and tests for association between the response ("Y" $=\ln T G$ ) and the $\mathrm{PCs}$ of the "X" variables that explain at least $80 \%$ of their total variation. This method takes advantage of the correlation structure within a gene region, and may increase power by reducing the dimensionality of the regressor set, such that more genes achieve significance even if their component CpG sites/SNPs are not detected in marginal regression.

For a given gene, let $\left\{P C_{1}^{s}, P C_{2}^{s}, \ldots, P C_{k}^{s}\right\}$ be the first $k$ PCs of the SNP variables associated with that gene, which explain $80 \%$ of their variation. Similarly, define $\left\{P C_{1}^{m}, P C_{2}^{m}, \ldots, P C_{l}^{m}\right\}$ as the first $l$ PCs of the methylation data. With this reduced data set, we fit the following regression models, including random effects for family:

$$
\begin{aligned}
\ln (\mathrm{TG}) \sim & P C_{1}^{s}+P C_{2}^{s}+\ldots+P C_{k}^{s}+\text { age } \\
& + \text { center }+ \text { sex } \\
\ln (\mathrm{TG}) \sim & P C_{1}^{m}+P C_{2}^{m}+\ldots+P C_{l}^{m}+\text { age } \\
& + \text { center }+\operatorname{sex}+P C 1^{G} \\
\ln (\mathrm{TG}) \sim & P C_{1}^{s}+\ldots+P C_{k}^{s}+P C_{1}^{m}+\ldots+P C_{l}^{m} \\
& + \text { age }+ \text { center }+\operatorname{sex}+P C 1^{G}
\end{aligned}
$$

We opted to use the first chromosome 11 global $P C^{G}$ of the methylation data as a measure of cell purity, as we found this produces a $\mathrm{CpG}$ test $p$ value 
Table 1 Top epigenetic signals for TGs (Model 1) detected in the GOLDN study data set $(n=995)$

\begin{tabular}{lllll}
\hline Mark name & Genes & Position & $p$ Value (Imer) & $p$ Value (Imekin) \\
\hline cg00574958 & CPT1A & $68,607,622$ & $6.52 \mathrm{E}-31$ & $1.23 \mathrm{e}-35$ \\
cg17058475 & CPT1A & $68,607,737$ & $1.61 \mathrm{E}-20$ & $1.31 \mathrm{e}-21$ \\
cg01082498 & CPT1A & $68,608,225$ & $2.21 \mathrm{E}-11$ & $2.85 \mathrm{e}-12$ \\
cg09737197 & CPT1A & $68,607,675$ & $7.30 \mathrm{E}-10$ & $9.34 \mathrm{e}-10$ \\
cg11376147 & SLC43A1 & $57,261,198$ & $2.51 \mathrm{E}-09$ & $7.53 \mathrm{e}-09$ \\
cg26989316 & CPT1A & $68,607,257$ & $1.90 \mathrm{E}-08$ & $7.56 \mathrm{e}-09$ \\
cg12556569 & APOA5 & $116,664,039$ & $2.25 \mathrm{E}-08$ & $4.63 \mathrm{e}-10$ \\
cg00264754 & LRRC4C & $40,136,810$ & $9.30 \mathrm{E}-08$ & $3.39 \mathrm{e}-07$ \\
\hline
\end{tabular}

distribution close to that expected under the null hypothesis. In each of the 3 models [models (2), (3), and (4); fitted via $\mathrm{R}$ function $l m e r$, a global Wald test is performed on the coefficient estimates $\hat{\beta}$ of the SNP and/or CpG PC terms. Model (4) is designed to assess the combined contribution of the $\mathrm{CpG}$ and SNP PCs and determine whether the 2 sets of PCs make independent contributions. Although we limited testing to chromosome 11, to control the overall Type 1 error level, we specify a genome-wide significance threshold for testing. Counting approximately 20,000 to 30,000 genes (and thus tests) yields a threshold of $2 \times 10^{-6}$

\section{Integration of predictors at the gene-region}

To further differentiate the relative contribution of SNPs and CpG sites in model (4), we compute variance inflation factors (VIFs) for each PC as a means to identify multicollinearity among the variables in the joint regression model. High correlation between SNP and CpG components may be undesirable because it can inflate standard error estimates. The VIF in a linear regression is computed as $V I F_{i}=\left(1-R_{i}^{2}\right)^{-1}$, where $R_{i}^{2}$ is obtained by regressing the $i^{\text {th }}$ predictor on all the other predictors. In our case, as $P C$ s in each data set are orthogonal, the VIF for a SNP PC will be based on its correlation with all the CpG PCs, and conversely.

\section{Results}

\section{Single CpG testing}

We reproduced the original study associations [3] for chromosome 11 by fitting model (1) to \%methylation for each CpG. Eight CpG sites achieved significance ( $p$ value $<10^{-7}$ ) with the top 4 sites the same as those found in the GOLDN study in CPT1A (Table 1).

\section{Gene-region testing of CpG's and SNPs}

We fit models (2) to (4) to each gene region in turn, and test the corresponding global association hypotheses for CpG's and SNPs using generalized Wald tests. We detect gene CPT1A using gene-region testing, but in addition we find 2 other genome-wide significant regions: AP006216.5 using methylation data, and BUD13 using genetic data (Table 2). These gene-regions are in the same neighborhood that also contains APOA5, detected in the single $\mathrm{CpG}$ analysis reported in Table 1 (Fig. 1).

The integration of the 2 data types does not seem to improve the overall association signal: testing found the roughly the same gene set to be significant as in the separate epigenetic and genetic analyses, with 3 of the top 4 genes having larger $p$ values (see Tables 2 and 3). The examination of pairwise correlations between CpG and SNP PCs within gene regions suggests that this can be explained by relationships of higher order CpG PCs with SNP PCs, particularly for CPT1A and APOA5. APOA5 was affected in both epigenetic and genetic components. The gene BUD13 detected in the genetic SNP analysis dropped below the detection threshold after adding CpG data, most likely a consequence of the increase in model degrees of freedom. Remarkably, we observe little Spearman rank correlation between the epigenetic and genetic gene-region $p$ values across the 2615 gene regions.

To address multicollinearity in predictor integration, we fit a reduced model (4) to the 2 genes in Table 3 with high VIFs (Fig. 2) by sequentially dropping high VIF PCs, until no term remains with a VIF larger than 2 (this corresponds to excluding those $P C$ s with $\geq 50 \%$ variation explained by the other predictors). This removes SNP PC1 and PC2 from the CPT1A model,

Table 2 Gene-region testing applied to separate epigenetic and genetic regressions (Models 2 and 3; $n=717$ )

\begin{tabular}{|c|c|c|c|c|c|c|}
\hline \multirow[t]{2}{*}{ Gene } & \multicolumn{2}{|c|}{ Gene region (BP) } & \multicolumn{2}{|l|}{ Epigenetic } & \multicolumn{2}{|l|}{ Genetic } \\
\hline & Start & End & Degrees of freedom & $p$ Value & Degrees of freedom & $p$ Value \\
\hline CPT1A & $68,522,088$ & $68,611,878$ & 33 & $3.44 e-14$ & 5 & 0.436 \\
\hline AP006216.5 & $116,683,920$ & $116,684,719$ & 7 & $3.51 e-06$ & 4 & 0.045 \\
\hline BUD13 & $116,618,886$ & $116,643,704$ & 17 & 0.538 & 5 & $1.48 e-07$ \\
\hline APOA5 & $116,660,083$ & $116,663,136$ & 17 & $1.95 e-04$ & 6 & $1.47 e-05$ \\
\hline
\end{tabular}




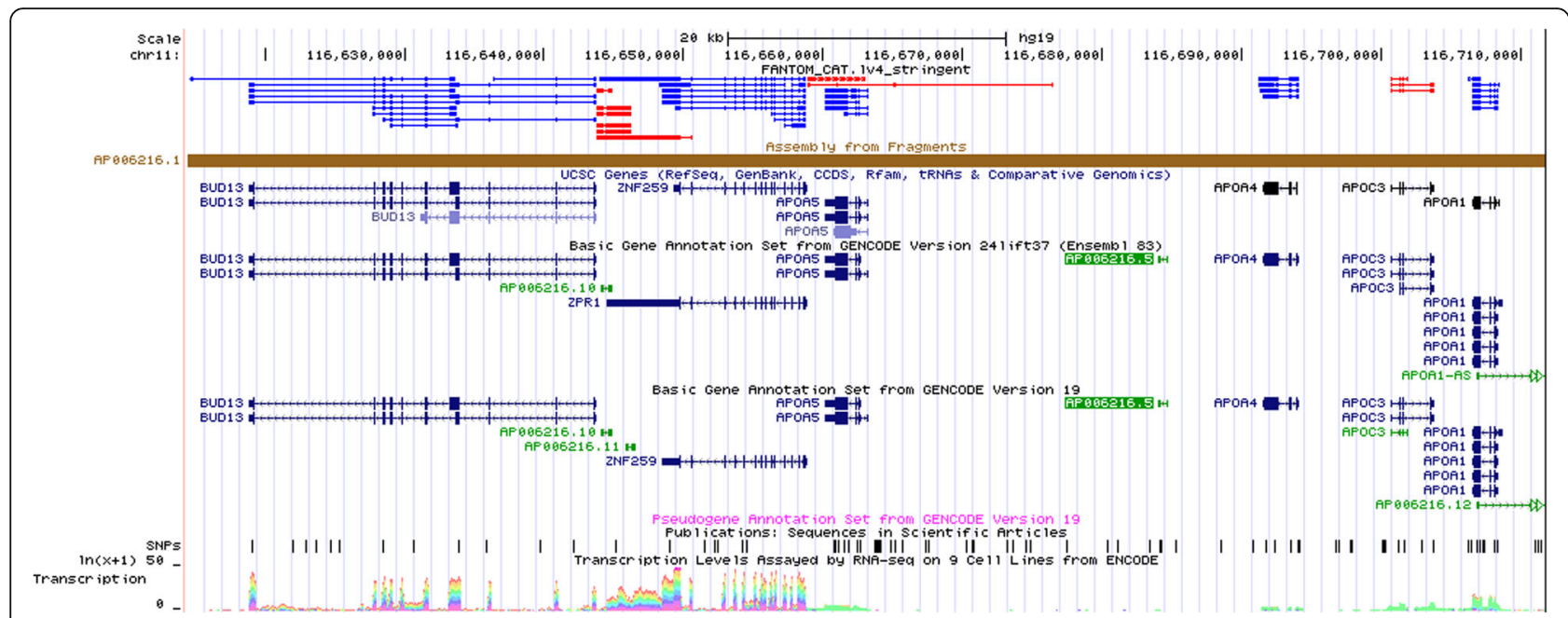

Fig. 1 Close-up view of the genetic region containing BUD13, APOA5, and AP006216.5 (from UCSC Genome Browser)

and CpG PC9 from the APOA5 model, (with VIFs for all remaining terms below 1.4), but does not improve the overall association signal ( $p$ values of $1.57 \mathrm{e}-13$ and 9.25e-04 for CPT1A and $A P O A 5$, respectively). For $A P O A 5$, the most highly collinear CpG $P C$ is also one of the strongest predictors of TG, suggesting that VIF pruning is not advisable for improving power, but can help clarify variable importance.

\section{Discussion}

In this contribution to GAW20, we investigate associations between a lipid phenotype (TG level) and epigenetic (methylation CpG sites) and/or genetic (SNP) markers. As an alternative to single-marker analysis, we apply a gene-region testing method based on multiple regressions of PCs summarizing CpG sites and SNPs in each gene region. The dimension reduction fraction achieved (number of PCs that explain at least $80 \%$ of data variability over the number of original variables) was often greater than $50 \%$, with greater data compression for larger genes, and SNP sets showing slightly more dimension-reduction capacity than $\mathrm{CpG}$ sites, despite having similar number of original variables (Fig. 3).

In separate and combined epigenetic and genetic regression analyses, we detected genome-wide significant gene-region $\mathrm{CPG}$ signals for the CPT1A gene reported in the original GOLDN study [3], as well as for 2 other genes. The 2 other gene regions lie within $50 \mathrm{~kb}$ of a single significant $\mathrm{CpG}$ detected in our single-variable $\mathrm{CpG}$ analysis, which suggests that this entire region harbors signals of association with TGs. For the CPT1A gene, the epigenetic component clearly leads the results, with no detectable genetic signal. Associations detected with AP006216.5, BUD13, and APOA5, all located in a different region of chromosome 11, also included epigenetic and/or genetic components. For AP006216.5, the epigenetic component leads the overall association, with an independent nominal genetic component. In contrast, for $B U D 13$, the genetic component is the sole contributor. For the APOA5 gene, which is located midway between AP006216.5 and BUD13, there is suggestive genome-wide association resulting from both epigenetic and genetic components, which are not independent, and we find evidence for relationships between certain CpG PCs and SNP PCs. Notably, $A P O A 5$ is a known genetic determinant of TG variation, and recent data points to joint genetic and epigenetic regulation of TG [6].

We attempt to increase power in the combined epigenetic and genetic regression using VIFs to eliminate multicollinearity among predictors. This produces 2 sets of regressors that are approximately orthogonal,

Table 3 Gene-region testing applied to integrated epigenetic-genetic regressions (Model 4; $n=717$ )

\begin{tabular}{|c|c|c|c|c|c|c|c|}
\hline \multirow[t]{2}{*}{ Gene } & \multirow{2}{*}{$\begin{array}{l}\text { BP Start } \\
\text { Start }\end{array}$} & \multicolumn{2}{|l|}{ Epigenetic } & \multicolumn{2}{|l|}{ Genetic } & \multicolumn{2}{|l|}{ Epigenetic + Genetic } \\
\hline & & Degrees of freedom & $p$ value & Degrees of freedom & $p$ Value & Degrees of freedom & $p$ Value \\
\hline CPT1A & $68,522,088$ & 33 & $2.47 e-14$ & 5 & 0.276 & 38 & $9.44 e-14$ \\
\hline AP006216.5 & $116,683,920$ & 7 & $2.62 e-06$ & 4 & 0.034 & 11 & $1.16 e-06$ \\
\hline BUD13 & $116,618,886$ & 17 & 0.711 & 5 & $7.13 e-07$ & 22 & $2.23 e-04$ \\
\hline APOA5 & $116,660,083$ & 17 & 0.088 & 6 & 0.064 & 23 & $7.19 e-05$ \\
\hline
\end{tabular}




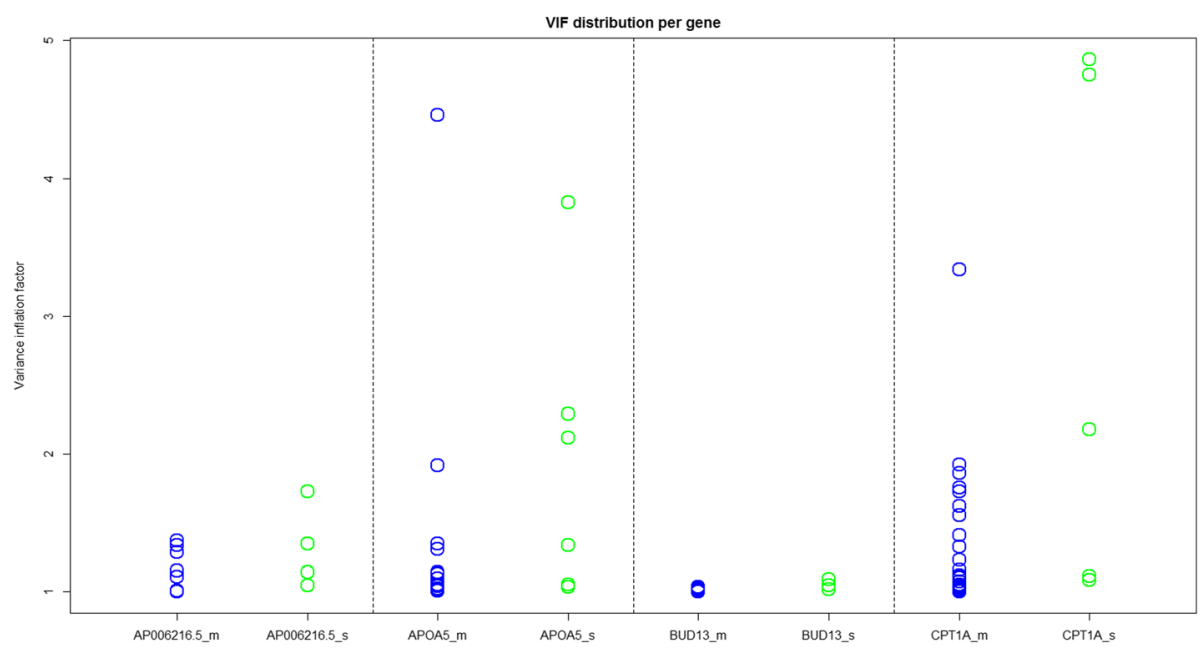

Fig. 2 Plots of VIFs for each of the top genes, corresponding to CpG PCs (blue) and SNP PCs (green)

facilitating evaluation of independent contributions of SNP- and CpG-based PCs, but this approach does not strengthen association signals in the combined regression. We speculate that this may be partly because the $P C$ s which are highly correlated between data types are likely to share causal etiology, so excluding them reduces power; and partly because most $P C$ s are largely uncorrelated, and the VIF approach does not eliminate these PCs. Our recommendation for future studies is that Gauderman's method works well at the gene level for separate analysis of both genetic and epigenetic data types, and integration of the 2 data sources, with assessment of their intercorrelation, can give further insight.

\section{Conclusions}

Using a gene-region testing approach that effectively reduced predictor dimensionality, we recovered the gene $C P T 1 A$ as having significant association between methylation and TG levels. In addition we identified 2 other genes that were not detected in the single $\mathrm{CpG}$ analysis: gene $B U D 13$, genetically significant, and region AP006216.5, epigenetically significant. In integration of the genetic and methylation data types when testing for association with TG levels at the gene level, although we found no evidence of improvement in association signal strength over separate analyses, use of a combined model helps clarify the relative contribution of epigenetic and genetic components.
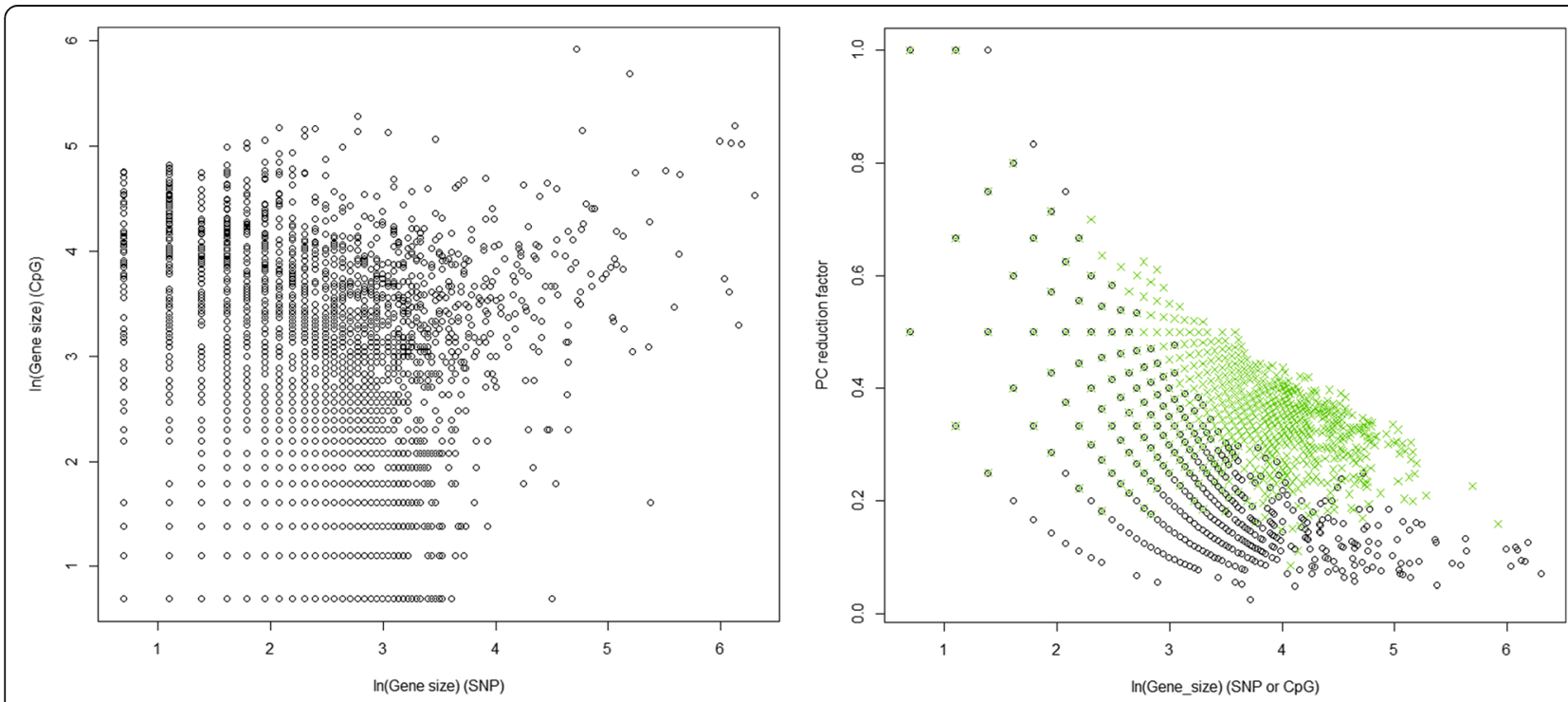

Fig. 3 Left: Gene sizes (log scale) in SNPs versus CpGs. Right: Fraction of dimension reduction achieved by applying Gauderman's method to SNPs (black circles) and CpGs (green Xs) on chromosome 11 


\section{Funding}

Publication of this article was supported by NIH R01 GM031575. This work was supported by funding from the Canadian Institutes of Health Research (project grant to SBB, STAGE training award to OEG) and a GAW20 travel award to RGR.

\section{Availability of data and materials}

The data that support the findings of this study are available from the Genetic Analysis Workshop (GAW), but restrictions apply to the availability of these data, which were used under license for the current study. Qualified researchers may request these data directly from GAW.

\section{About this supplement}

This article has been published as part of BMC Proceedings Volume 12 Supplement 9, 2018: Genetic Analysis Workshop 20: envisioning the future of statistical genetics by exploring methods for epigenetic and pharmacogenomic data. The full contents of the supplement are available online at https:// bmcproc.biomedcentral.com/articles/supplements/volume-12-supplement-9.

\section{Authors' contributions}

RGR, OEG, and SBB designed the overall study. RR and JM conducted statistical analyses. OEG supplied gene definitions. RR participated in the workshop and drafted the manuscript. SBB revised the manuscript for critical content. All authors read and approved the final manuscript.

\section{Ethics approval and consent to participate}

Not applicable.

\section{Consent for publication}

Not applicable.

\section{Competing interests}

The authors declare that they have no competing interests.

\section{Publisher's Note}

Springer Nature remains neutral with regard to jurisdictional claims in published maps and institutional affiliations.

\section{Author details}

${ }^{1}$ Lunenfeld-Tanenbaum Research Institute, Sinai Health System, 600

University Ave, Toronto, ON M5G 1X5, Canada. ${ }^{2}$ Dalla Lana School of Public Health, University of Toronto, 155 College St, Toronto, ON M5T 3M7, Canada.

Published: 17 September 2018

\section{References}

1. Yoo YJ, Sun L, Poirier JG, Paterson AD, Bull SB. Multiple-linear-combination regression tests for common variants adapted to linkage disequilibrium structure. Genet Epidemiol. 2017:41(2):108-21.

2. Gauderman WJ, Murcray C, Gilliland F, Conti DV. Testing association between disease and multiple SNPs in a candidate gene. Genet Epidemiol. 2007;31(5):383-95.

3. Irvin MR, Zhi D, Joehanes R, Mendelson M, Aslibekyan S, Claas SA, Thibeault KS, Patel N, Day K, Jones LW, et al. Epigenome-wide association study of fasting blood lipids in the genetics of lipid lowering drugs and diet network study. Circulation. 2014:130(7):565-72.

4. Gencode. http://www.gencodegenes.org/

5. LocusZoom. Version 1.3. https:/statgen.sph.umich.edu/locuszoom/download/

6. Oliva I, Guardiola M, Vallvé JC, Ibarretxe D, Plana N, Masana L, Monk D, Ribalta J. APOA5 genetic and epigenetic variability jointly regulate circulating triacylglycerol levels. Clin Sci. 2016;130(22):2053-9.

Ready to submit your research? Choose BMC and benefit from:

- fast, convenient online submission

- thorough peer review by experienced researchers in your field

- rapid publication on acceptance

- support for research data, including large and complex data types

- gold Open Access which fosters wider collaboration and increased citations

- maximum visibility for your research: over $100 \mathrm{M}$ website views per year

At BMC, research is always in progress.

Learn more biomedcentral.com/submissions 